\title{
ANÁLISIS DEL ESTADO FÍSICO Y PSICOLÓGICO DE LA POBLACIÓN ESCOLAR DEL CANTÓN CENTRAL DE HEREDIA, COSTA RICA
}

\section{ANALYSIS OF THE PHYSICALAND PSYCHOLOGICAL PROFILE OF THE SCHOOL POPULATION OF THE CENTRAL DISTRICT OF HEREDIA, COSTA RICA}

\author{
Emmanuel Herrera-González ${ }^{1}$ \\ Maria Morera-Castro ${ }^{2}$ \\ Gerardo Araya Vargas ${ }^{3}$ \\ Héctor Fonseca-Schmidt ${ }^{4}$ \\ María del Pilar Vargas Ramírez ${ }^{5}$ \\ Universidad Nacional, Costa Rica \\ Escuela de Ciencias del Movimiento Humano y Calidad de Vida, \\ Universidad Nacional, Costa Rica \\ emmanuel.herrera.gonzález@una.cr
}

\begin{abstract}
Resumen
El propósito del estudio fue establecer un perfil físico y psicológico de la población escolar de II Ciclo. De un total de 1.080 participantes, 318 son de cuarto grado, 418 de quinto y 344 de sexto, con edades entre 9 y 15 años (edad promedio de $11.10 \pm 1.07$ ). Para establecer el IMC se midió la talla y el peso. Para los niveles de actividad física se utilizaron dos instrumentos de auto-reporte: Cuestionario Pictórico de Actividad Física para la Población Infantil y el Cuestionario de Actividad Física para Niños y Niñas Mayores. La prueba "Progressive Aerobic Cardiovascular Endurance Run" evalúa el nivel de condición física de población infantil y juvenil. Las variables psicológicas se estudiaron mediante Cuestionario BIA y Cuestionario LAWSEQ. Según clasificación del Centro de Control y Prevención de Enfermedades (CDC), el nivel de sobrepeso y obesidad es de un 28.8\% y de bajo peso de $4 \%$. En la capacidad aérobica (VO2 máx), un $77.7 \%$ se encuentra en una zona saludable para su edad. Los niños se perciben físicamente más activos que las niñas. Con respecto a la autoestima, se encontró que un $35 \%$ muestra niveles bajos y solo un $10 \%$ presenta un nivel de autoestima alto. En imagen corporal la tendencia es ver la imagen ideal y futura más delgados/as.Estos resultados sirven como base científica para futuras investigaciones que tengan como objetivoel bienestar de la población escolar, tomando en cuenta el ligamen existente entre el área física y aspectos psicológicos en la niñez.
\end{abstract}

Palabras clave: niñez, salud, actividad física, imagen corporal, autoestima

\begin{abstract}
The purpose of this study was to establish a physical and psychological profile of fourth, fifth, and six graders. The study included a total of 1080 participants, specifically 318 fourth graders, 418 fifth graders and 344 sixth gradersbetween the ages of 9-15 years (mean age 11.10 \pm 1.07 ). Size and weight were measured to establish BMI. Two self-report instruments were used to establish levels of physical activity: Pictorial Questionnaire of Children's Physical Activity and Physical Activity Questionnaire for Older Boys and Girls. The PACER (Progressive Aerobic Cardiovascular Endurance Run) Test evaluates children and teenagers' fitness level. Psychological variables were studied using the BIA Questionnaire and Lawrence's Self-Esteem
\end{abstract}


Questionnaire (LAWSEQ). According to the classification by the Center for Disease Control and Prevention (CDC), the level of overweight and obesity is $28.8 \%$ while the level of underweight is $4 \%$. As for aerobic capacity (VO2max) $77.7 \%$ is in a healthy level for their age. Boyssee themselves more physically active than girls see themselves.Regarding self-esteem, $35 \%$ show low levels and only $10 \%$ have high self-esteem. As far as body image, the tendency of the ideal and future image is to be thinner.These results serve as a fundamental scientific basis for future research aimedat improving the health and well-being of the school population, taking into account the relationship between body image and children's psychological aspects.

Key Words: childhood, health, physical activity, body image,self-esteem.

\section{Introducción}

En la actualidad el desarrollo de tecnologías de la información, las comunicaciones y los medios de transporte han descendido el nivel de actividad física en la vida cotidiana, con consecuencias asociadas al sedentarismo, la obesidad y trastornos relacionados con la salud y la alimentación (López-Sánchez, González-Víllora y Díaz-Suárez, 2016). En particular, la población escolar de hoy presenta una tendencia a poseer hábitos alimentarios inadecuados y un estilo de vida sedentario que origina una alta prevalencia de niveles de sobrepeso y obesidad, patologías que pueden ocasionar problemas físicos y psicosociales en las primeras etapas de la vida, las cuales van a repercutir posteriormente en la etapa adulta (Zapata, Soriano, Márquez, López y González, 2015). Al considerarse la obesidad como de etiología multicausal, las investigaciones sobre la misma pueden tener diferentes enfoques: genético, nutricional, metabólico, psicológico, social, entre otros (Romero, Márquez-Rosa, Bernal, Camberos y De Paz, 2014).

Investigaciones realizadas por Cándido et al. (2012), De Piero, Rodríguez, González y López (2014), Fajardo y Arango (2012) y Trescastro-López, Trescastro- López y GalianaSánchez (2014) han constatado el aumento en los problemas de salud, referentes al sedentarismo, sobrepeso y la obesidad infantil en Latinoamérica y enEspaña, y destacan en esta tendencia una deficiente actividad física, que conducen a que sean las enfermedades de la nueva era.

En el ámbito costarricense, Ureña, Araya, Sánchez, Salas y Blanco (2010) estudiaron a 638 estudiantes (289 hombres y 349 mujeres) de la provincia de Guanacaste, con una edad media de 13.03 años, encontrando una prevalencia de sobrepeso-obesidad de un 13,9\% (según criterios del $\mathrm{CDC}$ ), así como una importante prevalencia de actividades sedentarias. Como contraparte, Fajardo y Arango (2012) plantean que son las intervenciones para la salud basadas en el comportamiento las más acertadas para la prevención de riesgo cardiovascular, pues se ha demostrado un incremento en la actividad física y la adopción de mejores hábitos alimentarios, en niños y adolescentes; esto corroborado por investigaciones recientes(López-Sánchez, Borrego-Balsalobre y Díaz-Suárez, 2013; Borrego-Balsalobre, López- Sánchez y Díaz Suárez, 2015; López-Sánchez, Nicolás-López \& Díaz-Suárez, 2016). 
Ahora bien, es importante considerar que aparte del estado físico de la niñez, existen otras áreas del desarrollo que son importantes de comprender para el bienestar de esta población. Entre ellos están los factores psicológicos, dentro de los cuales destacan la percepción de la imagen corporal y la autoestima, por los graves efectos psicológicos y de socialización que generan en la población infantil (Romero et al., 2014). En los primeros años de la infancia se define una autorepresentación mental del tamaño, figura y de la forma del cuerpo en general y de sus partes; es decir, cómo ve su propio cuerpo cada persona y cómo cree que los demás lo ven, ya que la imagen corporal está impregnada de valoraciones subjetivas, y está determinada socialmente, formada por diferentes componentes: perceptual, cognitivo, afectivo y conductual (López-Sánchez, López-Sánchez y Díaz-Suárez, 2015).

El trastorno de la imagen corporal es una condición que provoca malestar subjetivo, incidiendo negativamente en las relaciones sociales y en el desempeño académico, llevando al aislamiento y manifestándose en conductas obsesivo-compulsivas y trastornos alimentarios, con posibles orígenes en la preadolescencia (Romero et al., 2014). Una distorsión en tal percepción puede generar en la niñez comportamientos de riesgo para su salud (Brannon y Feist, 2010). Ortega, Muros, Palomares, Martín y Cepero (2014) y Trujano, Nava, de Gracia, Limón, Alatriste y Merino (2010) comprobaron que los estereotipos de delgadez extrema impactan más a las niñas que a los varones.

Por su parte, Mérida, Serrano y Tabernero (2015) plantean que la autoestima no es observable directamente, siendo necesario inferirla a partir de la conducta o basarse en informes personales acerca de lo que cada quien piensa de sí mismo; por lo que, autodescripciones orales o escritas, entrevistas o cuestionarios son instrumentos recomendados por estudios de autoestima y autoconcepto. En la presente investigación se utilizó un cuestionario de autoestima pertinente para la población infantil costarricense.

La práctica de la actividad física ha reportado efectos positivos en el autoconcepto, autoestima y autoeficacia de las personas (Borrego-Balsalobre, López-Sánchez y Díaz-Suárez, 2012; Borrego-Balsalobre, López-Sánchez y Díaz-Suárez, 2014; Brannon y Feist, 2010). Actualmente, la tendencia conlleva a realizar investigaciones de temas relacionados con la salud infantil $\mathrm{y}$, específicamente, de la obesidad; sin embargo, existe una faltante de estudios que contemplen una visión más integral y holística de la salud, que incluyan en sus variables todos los factores que la componen. En Costa Rica se han invertido pocos y aislados esfuerzos por conocer, comprender, caracterizar y contextualizar el perfil de la multiplicidad de factores relacionados con la salud, incluyendo factores genéticos, nutricionales, influencias del medioambiente, socialización, aspectos familiares, culturales socioeconómicos, historia médica y de otros acontecimientos en poblaciones infantiles, por lo que este estudio tiene como propósito realizar un análisis descriptivo del estado físico y psicológico de la población infantil herediana que asiste a la educación primaria pública. 


\section{Metodología}

\section{Participantes}

La muestra probabilística aleatoria se obtuvo de las listas de las 24 escuelas del Ministerio de Educación Pública ubicados en el cantón central de Heredia, agrupados por escuela, grado y sexo;3.664 estudiantes matriculados encuarto, quinto y sexto grado conforman la población de estudio. Para seleccionar las escuelas que conformaron la muestra de este estudio se estableció un punto de corte 232 estudiantes de II Ciclo matriculados por escuela. De este corte se estableció que doce escuelas cumplían con esta condición, de las cuales diez accedieron a participar. Sin embargo, durante el transcurso de la recolección de datos se presentaron algunos inconvenientes de participación, por lo que se procedió a contactar dos escuelas más para aumentar la muestra de estudio. Un total de 1080 participantes conforman la muestra de investigación, de los cuales 318 son de cuarto grado, 418 de quinto grado y 344 de sexto grado, con edades de 9 a 15 años (edad promedio de $11.10 \pm 1.07$ ).

\section{Instrumentos}

Las variables físicas de esta investigación contemplan: índice de masa corporal, niveles de actividad física y la capacidad aeróbica. A continuación, se describe cada instrumento utilizado por variable.

\section{Índice de masa corporal}

Para establecer el índice de masa corporal (IMC) se midió la talla y el peso de cada participante. La talla se midió con cinta métrica. Al niño/a se le pidió que permaneciera de espalda contra una pared; seguidamente, la altura se registró en centímetros. El peso fue medido a través de una balanza modelo Tanita Body Composition Monitor IRONMAN BC-554. Al niño/a se le solicitó que se subiera en la balanza sin medias ni calzado, el peso fue registrado en kilos, siguiendo el protocolo establecido por la Organización Mundial de la Salud (OMS, 2015a) en el método STEPwise de vigilancia. Para calcular el IMC de cada persona se utilizó la herramienta de cálculo para población infantil y juvenil (2 a 19 años) ofrecida por el Centro de Control y Prevención de Enfermedades (CDC) de los Estados Unidos en su sitio web, la cual estima el IMC por percentil de edad, según los cuadros de crecimiento por edad de CDC, a partir de la fecha de nacimiento, el día de medición, el peso y la talla de la persona (Centro de Control y Prevención de Enfermedades, 2015).

\section{Nivel de actividad física}

Para establecer el nivel de actividad física de cada persona participante se utilizaron dos instrumentos de auto-reporte, los cuales se describe a continuación. 


\section{Cuestionario Pictórico de Actividad Física para la Población Infantil (C-PAFI)}

Es una medida de auto-reporte de los niveles de actividad física en población infantil de 7 a 13 años, con cinco preguntas cerradas de diferentes momentos de la actividad del niño/niña. Estos se identificaron con un dibujo que representa cuatro estadios (sedentario, poco activo, activo, muy activo) durante la última semana, con una versión para niño y otra para niña. El promedio general representa el nivel de actividad física de cada participante (1 a 4 puntos), siendo cuatro el estadio más activo. Cuenta con una validez por expertos, además de la validez de criterio entre medidas de auto-reporte PAQ-C y C-PAFI, con una correlación de Pearson significativa moderada ( $r=0.59$, $p \leq .001$ ) y una correlación de Spearman moderada entre PAQ-C y el podómetro (rho $=0.368, p$ $=.049$ ) durante la semana. Además de una confiabilidad por medio del alfa de Cronbach de 0.56 (Morera-Castro, Herrera-González, Araya-Vargas y Jiménez-Díaz, in press).

\section{Cuestionario de Actividad Física para Niños y Niñas Mayores (PAQ-C)}

En inglés, "Physical Activity Questionnaire for Older Children”, con siglas PAQ-C (Crocker, Bailey, Faulkner, Kowalski \& McGrath, 1997). Es un instrumento indicado para niños y niñas con edades entre los 8 y 14 años. Consiste en una medida de autoreporte que proporciona una puntuación general de actividad física durante los últimos siete días, en diferentes situaciones y diferentes momentos del día. Nueve preguntas cerradas de selección múltiple con diferentes tipos de escala de Likert que se puntúan de uno a cinco,siendo cinco una persona activa. El PAQ-C, versión original en inglés, mostró una correlación con el rango de actividad $(\mathrm{r}=0,57)$, Leisure Time Exercise Questionanaire [LTEQ] $(\mathrm{r}=0,41)$, acelerómetro Caltrac $(\mathrm{r}=0,39)$, Entrevista de Recordatorio de Siete Días $[P A R](\mathrm{r}=0,46)$ y la prueba Canadiense de fitness [test de pasos] $(\mathrm{r}=$ 0,28) (Kowalski, Crocker y Faulkner, 1997). El PAQ-C demostró tener una adecuada confiabilidad test-retest $(\mathrm{r}=.75$ a .82$)$ y una validez razonable $(\mathrm{r}=.45$ a .53$)$ en comparación con las medidas objetivas de actividad física (Crocker et al., 1997; Janz, Lutuchy, Wenthe y Levy, 2008). Este instrumento ha sido validado al español por Martínez-Gómez et al. (2009) para adolescentes españoles. Para efectos de esta investigación se realizaron modificaciones culturales de las actividades físicas establecidas en la pregunta uno y se realizó una sustitución de aquellas palabras españolas (léxico) que no son utilizadas en Costa Rica por palabras autóctonas, para una mayor comprensión de las personas participantes, como lo recomiendan Moore, Hanes, Barbeau, Gutin, Treviño y Yin (2007).

\section{Capacidad aeróbica}

La prueba "Progressive Aerobic Cardiovascular Endurance Run" (con siglas en inglés PACER) evalúa el nivel de condición física (capacidad aeróbica) de la población infantil y juvenil. Esta prueba consiste en correr continuamente de un lado para el otro una distancia de 20 metros con un ritmo por etapa de un 1 minuto a un ritmo inicial de carrera de $8.5 \mathrm{~km}-\mathrm{h}-1$, y un incremento de velocidad de $0.5 \mathrm{~km} \mathrm{h-1}$ por etapa (Meredith y Welk, 2010), de acuerdo con un indicador reproducido por un CD. Un sonido indicará cuando la persona debe llegar al final de cada vuelta. Se registrará el número 
de vueltas que el niño/a logre realizar. La prueba finaliza cuando el niño/a en dos ocasiones no logre alcanzar la vuelta en el tiempo indicado. A partir del IMC y la cantidad de vueltas obtenidas por el niño/a se calcula, por medio de las tablas estándar establecidas por edad y sexo (Welk, Going, Morrow y Meredith, 2011), el VO2máx de cada persona, clasificándolas en saludables y en zonas de riesgo(Welk et al., 2011; Zhu, Mahar, Welk, Going y Cureton, 2011). Según Burns, Hannon, Allen y Brusseau (2014), el PACER cuenta con una precisión relativamente alta con respecto al modelo de la prueba de la milla corriendo/caminando, con un coeficiente de correlación fuerte $(r \geq 0.80, p \leq .001)$, un acuerdo en la zona de condición física de moderado a excelente con un kappa $>.40$ y un acuerdo $>.90$ y el Plot de Bland y Altman; para ambas pruebas arrogaron límites similares.

Los cuestionarios para medir las variables psicológicas se describen a continuación.

\section{Imagen corporal}

Cuestionario BIA (Evaluación de la Imagen Corporal), con nombre en inglés Body Image Assessment (BIA). Este permite obtener un índice indicativo de la percepción subjetiva de la imagen corporal real, ideal, social y futura, así como las discrepancias que el niño o la niña perciben entre sus yoes (real, ideal, social y futura). Esto por medio de cuatro preguntas, valoradas con una escala de siete figuras de niños y siete de adolescentes jóvenes y adultos de ambos sexos, que van desde una figura muy delgada (valor puntual de 1) hasta una figura con obesidad (valor puntual de 7), con un incremento puntual de 0.5 pts, las mismas establecidas según las curvas estándar de percentiles para el IMC en la niñez (Collins, 1991).

\section{Autoestima}

Cuestionario sobre Autoestima LAWSEQ, con nombre en inglés Lawrence Self-Esteem Questionnaire (LAWSEQ). Este instrumento valora el nivel de autoestima en la población infantil y la niñez, con base en la suma de características físicas y cognitivas de sí mismo. Cuenta con un total de 16 preguntas que valoran directamente la percepción sobre sí mismo. La fiabilidad interna de la escala se reporta de $\alpha=.76$ (Lawrence, 1981) y una aceptable validez externa con respecto al Coopersmith Self-Esteem Inventory $(r=0.73)$.

\section{Procedimiento}

Inicialmente se contactó al director regional del sector Heredia del Ministerio de Educación Pública; una vez obtenido su aval, se procedió a explicarles la investigación al director/a de cada escuela. Obtenidos los permisos de parte de ellos/as se contactó a las maestras/as de cuarto, quinto y sexto grado para explicarles en qué consistía la investigación y la importancia de la misma. Seguidamente, se repartió el consentimiento de la persona encargada y asentimiento para autorizar la participación de su hijo o hija en este estudio de acuerdo conlos criterios de bioética de la UNA. Una vez recolectados los consentimientos y asentimientos firmados por ambas personas, se realizaron 
tres visitas, en un lapso de tres semanas (una vista por semana). En una primera visita se procedió de manera individual a medir el peso y la talla, según los protocolos establecidos. En la segunda visita se midió la capacidad aeróbica y los cuestionarios de actividad física (C-CAFI y PAQ-C). En la tercera visita se procedió a aplicar los cuestionarios de autoestima y percepción de la imagen corporal. El niño o la niña respondieron los cuestionarios durante un día regular de clases, en presencia de su maestro/a de aula, brindando su nombre (sin apellidos), sexo, edad y grado.

\section{Análisis de datos}

Se tabularon y analizaron todos los datos utilizando el paquete estadístico SPSS 18.0. Se realizaron análisis de tipo descriptivo (media y desviación estándar) para todas las variables en estudio, por edad y sexo,talla, peso, IMC, PACER, C-PAFI, PAQ-C, imagen corporal y autoestima.Previamente, se realizó un análisis de frecuencia para detectar algún error o valor atípico. Asimismo, es importante mencionar que la cantidad de los datos no coinciden entre las variables dadas la fluctuación de participantes.

\section{Resultados}

En la Tabla 1 se puede observar la muestra por variable, así como el promedio, desviaciones estándares, valores mínimos y valores máximos de todas las variables estudiadas.

Tabla 1

Descripción de los promedios y desviaciones estándares por variable de estudio

\begin{tabular}{lccccc}
\hline \multicolumn{1}{c}{ Variables } & $n$ & Media & DE & Mínimo & Máximo \\
\hline Talla $(\mathrm{cm})$ & 944 & 144.91 & 8.81 & 123 & 173 \\
Peso $(\mathrm{kg})$ & 942 & 41.10 & 10.33 & 21.30 & 87.10 \\
IMC $\left(\mathrm{kg} / \mathrm{m}^{2}\right)$ & 942 & 19.36 & 3.57 & 11.80 & 34.50 \\
PACER & 284 & 24.38 & 12.33 & 2 & 64 \\
VO2max & 283 & 43.53 & 4.15 & 31.40 & 53.80 \\
PAQ-C & 741 & 2.87 & 0.66 & 0.18 & 4.93 \\
C-AFPI & 743 & 2.95 & 0.57 & 1 & 4 \\
Autoestima & 749 & 16.72 & 4.87 & 1 & 24 \\
IC real & 748 & 3.95 & 0.77 & 1 & 7 \\
IC ideal & 745 & 3.67 & 0.67 & 1 & 5.50 \\
IC social & 744 & 3.97 & 1.04 & 1 & 7 \\
IC futura & 742 & 3.60 & 0.66 & 1 & 5.50 \\
\hline
\end{tabular}

Nota: $\mathrm{IMC}=$ índice de masa corporal, IC= imagen corporal. 
Por su parte, se encontró que un 29\% (272) de la muestra presenta sobrepeso y obesidad, un $67 \%$ presenta un peso típico y un $4 \%$ (38) tiene bajo peso. Sin importar la edad, hay una tendencia en niños a mantener un IMC semejante; con excepción del joven de 15 años, cuyo IMC está por encima del promedio. Al contrario, las niñas tienden a incrementar su IMC conforme avanzan en edad (ver Tabla 2).

Tabla 2

Promedio y desviaciones estándar de la talla, peso e IMC de la muestra por edad y sexo

\begin{tabular}{lccccc}
\hline Sexo & Edad & $n$ & $\begin{array}{c}\text { Talla } \\
(\mathrm{cm})\end{array}$ & $\begin{array}{c}\text { Peso } \\
(\mathrm{kg})\end{array}$ & $\begin{array}{c}\text { IMC } \\
\left(\mathrm{kg} / \mathrm{m}^{2}\right)\end{array}$ \\
Niños & 9 & 26 & $135.28 \pm 5.47$ & $35.51 \pm 8.33$ & $19.25 \pm 3.65$ \\
& 10 & 130 & $139.55 \pm 6.92$ & $37.16 \pm 8.64$ & $18.96 \pm 3.31$ \\
& 11 & 146 & $144.75 \pm 7.39$ & $41.33 \pm 11.06$ & $19.52 \pm 4.10$ \\
& 12 & 128 & $148.23 \pm 8.50$ & $43.96 \pm 10.38$ & $19.83 \pm 3.41$ \\
& 13 & 23 & $151.35 \pm 9.13$ & $45.37 \pm 11.90$ & $19.40 \pm 3.50$ \\
& 14 & 9 & $155.11 \pm 9.14$ & $46.47 \pm 13.47$ & $19.23 \pm 5.00$ \\
& 15 & 1 & $173.00 \pm 0$ & $70 \pm 0$ & $23.40 \pm 0$ \\
& 9 & 36 & $136.68 \pm 5.94$ & $32.71 \pm 5.43$ & $17.53 \pm 2.86$ \\
& 10 & 126 & $140.38 \pm 7.76$ & $38.09 \pm 9.21$ & $19.16 \pm 3.39$ \\
& 11 & 165 & $146.63 \pm 7.92$ & $41.70 \pm 9.88$ & $19.22 \pm 3.61$ \\
& 12 & 120 & $150.04 \pm 6.49$ & $43.85 \pm 8.66$ & $19.37 \pm 3.07$ \\
& 13 & 23 & $152.65 \pm 7.74$ & $50.52 \pm 9.25$ & $21.65 \pm 3.52$ \\
& 14 & 9 & $153.08 \pm 6.97$ & $53.73 \pm 11.27$ & $22.82 \pm 4.08$ \\
\hline
\end{tabular}

Según lo observado en la Tabla 3, los resultados indican que existe una tendencia en los niveles de actividad en niños, según el C-AFPI y PAQ-C; en promedio se perciben moderadamente activos. Esto en contraposición a las niñas, cuya percepción del nivel de actividad física tiende a bajar durante los 10 a 13 años e incrementa nuevamente a los 14 años. En cuanto al VO2max, los niños concuerdan con la tendencia en sus niveles de actividad física; sin embargo, en las niñas el VO2max tiende a disminuir con la edad. Por su parte, según la clasificación establecida por Welk et al. (2011) y Zhu et al. (2011), un 77.7 \% (220) de la muestra se encuentra en una zona de VO2max saludable para su edad; sin embargo, un 22.3\% (64) necesita mejorar.

En el cuestionario C-AFPI se encuentra una serie de preguntas sobre la participación del niño/a en actividad física estructurada, frecuencia y duración, así como el medio de transporte más utilizado para ir a la escuela. Un total de 377 (50.9\%) niños y niñas pertenecen a algún tipo de agrupación deportiva o de danza, actividad que practican con mayor frecuencia de una a tres 
veces por semana (71.54\%), en sesiones de uno o más horas de práctica (74.73\%). El medio de transporte más utilizado es caminar a la escuela (294 personas), seguido por el bus o buseta (226 personas), el carro obtiene el tercer lugar de importancia (195 personas), siendo los medios menos usados la moto, con cinco, y la bicicleta, utilizada únicamente por dos personas.

Tabla 3

Características de nivel de actividad física y capacidad aeróbica de la muestra por edad y sexo

\begin{tabular}{lcccccc}
\hline Sexo & Edad & $n$ & C-AFPI & PAQ-C & $n$ & $\begin{array}{c}\text { PACER } \\
\text { (VO2max) }\end{array}$ \\
\hline Niños & 9 & 23 & $3.18 \pm 0.44$ & $3.12 \pm 0.71$ & 8 & $43.96 \pm 5.25$ \\
& 10 & 97 & $3.15 \pm 0.53$ & $3.15 \pm 0.65$ & 44 & $45.20 \pm 3.14$ \\
& 11 & 106 & $3.12 \pm 0.53$ & $3.00 \pm 0.70$ & 46 & $46.46 \pm 3.91$ \\
& 12 & 101 & $3.08 \pm 0.49$ & $2.97 \pm 0.72$ & 32 & $45.51 \pm 3.38$ \\
& 13 & 15 & $3.03 \pm 0.55$ & $3.02 \pm 0.46$ & 9 & $46.01 \pm 3.49$ \\
& 14 & 4 & $3.25 \pm 0.77$ & $2.75 \pm 0.57$ & 5 & $46.98 \pm 4.01$ \\
Niñas & 15 & & & & 1 & $45.20 \pm 0$ \\
& 9 & 28 & $3.02 \pm 0.52$ & $3.07 \pm 0.57$ & 9 & $42.33 \pm 3.22$ \\
& 10 & 98 & $2.81 \pm 0.61$ & $2.70 \pm 0.57$ & 31 & $41.78 \pm 3.97$ \\
& 11 & 124 & $2.75 \pm 0.57$ & $2.69 \pm 0.64$ & 55 & $41.46 \pm 3.15$ \\
& 12 & 95 & $2.74 \pm 0.54$ & $2.58 \pm 0.53$ & 31 & $40.97 \pm 3.10$ \\
& 13 & 13 & $2.86 \pm 0.55$ & $2.63 \pm 0.70$ & 9 & $38.90 \pm 2.56$ \\
& 14 & 4 & $3.4 \pm 0.51$ & $2.98 \pm 0.45$ & 3 & $38.23 \pm 2.40$ \\
\hline
\end{tabular}

Con respecto a las variables psicológicas, los resultados demuestran que los niveles de autoestima tienden a ser bajos en niños de 9 y 13 años, y las niñas de 10, 11 y 14 años (ver Tabla 4), esto representa un 35\% (264) de las personas participantes; un 55\% cuenta con un nivel de autoestima normal y solo un 10\% (71) presenta un nivel de autoestima alto.

En cuanto a la imagen corporal, los resultados establecen que el promedio fue mayor para los niños que para las niñas. Un patrón interesante se presenta en ambos sexos, en relación con la imagen corporal. Al preguntarles cómo se ven (imagen corporal real), la respuesta se agrupa en la imagen de una persona de peso normal. Sin embargo, el promedio de imagen corporal disminuye cuando se les pregunta la ideal y futura; es decir, se podría pensar que quieren verse más delgados/as. El promedio vuelve a aumentar en la percepción de cómo los ven las demás personas, siendo incluso en las niñas superior el promedio con respecto a su percepción de imagen real (ver Tabla 4). 


\section{Discusión}

Investigaciones dedicadas a determinar los niveles de sobrepeso y obesidad marcan una tendencia en el interés hacia este campo de la salud y el movimiento. Los resultados describen un aumento en el sobrepeso y la obesidad, no solo en personas adultas sino también en la niñez, evidenciando un gran problema social (Rivera et al. 2014; Mancipe et al. 2015). Estos resultados se ven reflejados en los datos encontrados en la presente investigación, donde un $28.8 \%$ de la población infantil valorada se encuentra en una clasificación de sobrepeso u obesidad, representando esto a 272 participantes, de un total de 840. Esto indica que la región en la cual se realizó el estudio no escapa a la problemática evidenciada por la OMS (2015b), que identifica a un 10\% de los niños y niñas en edad escolar con sobrepeso. Se concluye que el sobrepeso y la obesidad son problemas de salud que enfrenta la población en estudio y se considera que se deben realizar esfuerzos conjuntos entre los múltiples actores sociales para prevenir el aumento de esta epidemia en la población infantil. Sin embargo, tampoco se puede dejar de lado el $4 \%$ de la muestra que presenta bajo peso, ya que ambos extremos son perjudiciales para la salud de esta población.

Por otra parte, investigaciones como la de López et al. (2013) concluyen que la obesidad evaluada por el IMC y el porcentaje de grasa corporal se relaciona inversamente con la aptitud muscular y cardiovascular en escolares. Estudios recientes muestran que la capacidad cardiorrespiratoria es un potente indicador de salud futura enla infancia y adolescencia (ACSM, 2014; OMS, 2010). De acuerdo con los resultados del presente estudio, el $22.3 \%$ de la muestra valorada se encuentra en una zona de VO2max no saludable, la cual necesitan mejorar. A futuro, ellos podrían representar una población de adolescentes y adultos con potenciales problemas de salud, o bien, ser parte del $29.1 \%$ de niños y niñas con problemas de sobrepeso y obesidad. Estos datos son respaldados por un estudio longitudinal en el que participaron 598 niños y niñas estonios y suecos, quienes mostraban una alta capacidad cardiorrespiratoria durante la niñez, tendrían menor riesgo de desarrollar sobrepeso/obesidad durante la pubertad. Recientemente en un estudio con 700.000 adolescentes varones se observó que una mayor capacidad cardiorrespiratoria en la adolescencia se asociaba con menor riesgo de infarto de miocardio futuro (Högstrom, G., Nordström, A., \& Nordström, 2014). De aquí la importancia de mejorar la capacidad cardiorrespiratoria encontrada en esta muestra, especialmente en las niñas de 10 años en adelante.

En cuanto al nivel de actividad física de la muestra, en estudios se evidenció que los niños se perciben como personas moderadamente activas, mientras que en las niñas, a partir de los 10 años y hasta los 13, su nivel de actividad física se tiende a percibir como inferior a los años anteriores. Esto concuerda con investigaciones como Álvarez, (2016); Calahorro, Torres, López, Álvarez (2014) y Hallal et al. (2012).

Además, es importante señalar que poco más de la mitad de esta población tiene como hábito la práctica regular de una actividad sistemática, deportiva o de danza, y que un 58.21\% de utilizaba como medio de transporte para ir o venir de la escuela un vehículo automotor. Investigaciones han demostrado que la participación infantil en programas estructurados $\mathrm{y}$ 
guiados profesional y adecuadamente, en donde se favorezca el desarrollo de actitudes positivas hacia la actividad física, es necesaria para mejorar su autopercepción y para que adopten hábitos regulares de actividad física, y combatir así, más efectivamente, la epidemia de obesidad en niños, niñas y adolescentes (Tsang, Kohn, Chow y Singh, 2013). Por tanto, se concluye que es importante redoblar esfuerzos en la promoción de la práctica de actividad física en esta población, siguiendo las recomendaciones internacionales vigentes (ACSM, 2014; OMS, 2010); especialmente en niñas de más de 10 años de edad, ya que, según lo encontrado, son quienes reportanniveles menores de actividad física.

Con respecto a las variables psicológicas estudiadas, se encontró que los niveles de autoestima de la población infantil valorada tienden a ser bajos y solo un $10 \%$ presentó un nivel de autoestima alto. Romero et al. (2014) describen a la obesidad como generadora de problemas que dificultan la calidad de vida, inscrita en una sociedad obeso-fóbica que conduce a quien presenta este padecimiento a una desvalorización de la persona de manera persistente y cotidiana. Agregan, además, que un historial de fracasos en el seguimiento de regímenes alimentarios estrictos hace que en la personalidad frágil del niño se arraiguen los rasgos de depresión, ansiedad, angustia, baja autoestima y, en ocasiones, trastornos compulsivos alimentarios. En el sentido contrario, Raimann y Verdugo (2012) reportan que cuando los niños y niñas experimentan cambios físicos, se presentan mejoras en la autoestima.

En relación con la percepción de imagen corporal, si bien los resultados de esta investigación demuestran una tendencia mayor en la selección de la imagen de una persona de peso normal, el promedio de imagen corporal tendió a disminuir cuando se les preguntaba a las personas participantes por su imagen ideal y futura, por lo cual se podría pensar que niños y niñas quieren verse más delgados/as.

Otras investigaciones referidas por Romero, et al. (2014) han realizado comparaciones entre infantes y preadolescentes con adolescentes, encontrando que con el paso de los años, el problema de la autopercepción es aún mayor; el 55\% de las niñas de 7 a 12 años desea estar más delgadas, en la adolescencia el porcentaje asciende hasta el $80 \%$, presentándose en esta etapa también niveles de autoestima más bajos. La problemática surgida en torno a la imagen corporal se relaciona con la gran cantidad de alteraciones que le provoca al niño, principalmente en su autoestima (Romero, et. al., 2014), describiendo los autores que, si a una persona no le gusta su cuerpo, difícilmente le gustará la persona que vive en él, dificultando con ello la apreciación de cualidades y/o de destreza e inteligencia independientes al aprecio que tenga por su cuerpo.

En este estudio, un hallazgo importante fue la percepción de imagen corporal por parte las demás personas reportada por los sujetos, quienes ellos consideran tienden a percibirlos con mayor peso, siendo incluso en las niñas superior su valoración a la del promedio, con respecto a su percepción de imagen real. Coincide esta información con la de estudios que concluyen que la obesidad genera un impacto psicológico en la personalidad de la niñez que la padece. Esto es muy significativo, ya que mantiene la convicción irracional de ser "anormal" y el miedo a las 
relaciones con los demás (Romero, et al. 2014). Estos resultados deben profundizarse en futuros estudios, dado el ligamen que se ha demostrado entre la percepción de la imagen corporal y las conductas alimentarias, las cuales repercuten en la salud y calidad de vida de las personas y cuya base se forja en la infancia. La presente investigación coincide con el llamado que se hace desde el estudio de Romero et al. (2014), en el que se concluye que el ejercicio físico en niñosy niñas con obesidad parece tornarse más importante en los cambios provocados en la autoimagen que en la imagen corporal, en la creación de hábitos de ejercicio en estos niños e instan a realizar más investigaciones sobre los mecanismos a través de los cuales el ejercicio físico mejora la autoimagen, aunque no provoque cambios constatables en el cuerpo.

Hay que recordar que la actividad física es algo innato en la población infantil y la niñez, por lo que se requiere conservarla, promoverla y fortalecerla, sobre todo en esta época, en la cual el sedentarismo es el estilo de vida predominante, y se hace necesario diseñar e implementar programas y acciones que lo disminuyan. Por tanto, es importante redoblar esfuerzos en la promoción de la práctica de actividad física en esta población, de acuerdo con las recomendaciones internacionales vigentes.

Estos resultados son un primer acercamiento a la realidad nacional en materia de niñez y adolescencia; sin embargo, se deben continuar realizando investigaciones que profundicen el estado físico y psicológico, así como determinar los factores que mayor influencian en el mejoramiento de su bienestar y calidad de vida. Asimismo, y debido al alto bombardeo sobre la imagen corporal ideal que sufren estas poblaciones desde edades tempranas, es importante seguir realizando investigaciones relacionadas con la percepción de la imagen corporal y las conductas alimentarias, las cuales repercuten en la salud y calidad de vida de las personas y cuya base se forja en la infancia.

Mucho trabajo a nivel de investigación queda por realizar en cuanto a la población infantil, niñez y adolescencia en Costa Rica, no solo a nivel descriptivo sino a nivel comparativo y asociativo, por lo que se insta a la población científica, profesores, maestros y comunidad en general, a volcar sus ojos hacia este grupo, en beneficio de formar futuros ciudadanos y ciudadanas saludables. Como bien lo establece la OMS, son las escuelas un medio fundamental para el desarrollo de hábitos de vida saludables y la realización de programas de prevención, al reconocer que la edad escolar es un periodo importante para promover y consolidar habilidades en todas las áreas del desarrollo, para reafirmar hábitos de alimentación saludables y para que los niños y las niñas alcancen una excelente calidad de vida.

\section{Referencias bibliográficas}

Álvarez, C. Entendiendo los factores que determinan la actividad física en el entorno escolar desde la perspectiva de los niños y niñas. MHSALUD: Revista en Ciencias del Movimiento Humano y Salud, 13 (1). doi: http://dx.doi.org/10.15359/mhs.13-1.2 
American College of Sports Medicine [ACSM] (2014). ACSM's guidelines for exercise testing and prescription (9 ed.). Philadelphia / Baltimore: Wolters Kluwer / Lippincott Williams \& Wilkins.

Borrego-Balsalobre, F. J., López-Sánchez, G. F. \& Díaz Suárez, A. (2012). Physical condition influence in self-concept of a teens group of Alcantarilla town. Cuadernos de Psicología del Deporte, 12 (2), 57-62.

Borrego-Balsalobre, F. J., López-Sánchez, GF. \& Díaz-Suárez, A. (2014). Relationships between physical fitness and physical self-concept in Spanish adolescents. PROCEDIA: Social and Behavioral Sciences, 132, 343-350. doi: http://dx.doi.org/10.1016/j.sbspro.2014.04.320

Borrego-Balsalobre, F. J., López Sánchez, G. F. \& Díaz Suárez, A. (2015a). Effects of a vigorous physical activity program in the endurance of primary school children. ATHLOS: International Journal of Social Sciences of Physical Activity, Game and Sport, 8, 31-46.

Borrego-Balsalobre, F. J., López Sánchez, G. F. \& Díaz Suárez, A. (2015). Influence of a vigorous physical activity program on cholesterol level of Primary schoolchildren. AGON: International Journal of Sport Sciences, 5 (2), 60-71. Recuperado de: http://agonjournal.com/wp-content/ uploads/2016/03/AGON_5_2_Completo.pdf

Brannon, L.\& Feist, J. (2010). Health psychlogy: An introduction to behavior and health. Belmont: Wadsworth.

Burns, R. D., Hannon, J. C., Brett M. Allen, B. M. \& Brusseau. T. A. (2014). Convergent validity of the One-Mile Run and PACER VO2max prediction models in middle school students. SAGE Open, 4 (1), 1-8. doi: http://dx.doi.org/10.1177/2158244014525420

Cándido, A., Alosta, J., Oliveira, C., Freitas, R., Freitas, S. \& Machado, G. (2012). Anthropometric methods for obesity screening in schoolchildren. Nutrición Hospitalaria, 27 (1) 146-153. Recuperado de: https://www.ncbi.nlm.nih.gov/pubmed/22566314

Calahorro, F., Torres-Luque, G., López-Fernández, I.y Álvarez, E. (2014). Niveles de actividad física y acelerometría: recomendaciones y patrones de movimiento en escolares. Cuadernos de Psicología del Deporte, 14(3), 129-140. Recuperado de: http://scielo.isciii.es/ scielo.php?script=sci_arttext\&pid=S1578-84232014000300014\&lng=es\&tlng=es.

Centro de Control y Prevención de Enfermedades [CDC] (2015). Calculadora del índice de masa corporal para la población infantil y juvenil. Recuperado de:https://nccd.cdc.gov/dnpabmi/ Calculator.aspx

Collins, M.E. (1991). Body figure perceptions and preferences among preadolescent children. International Journal of Eating Disorders, 10, 199-208. doi: https://doi.org/10.1002/1098108X(199103)10:2<199::AID-EAT2260100209>3.0.CO;2-D 
Crocker, P. R. E., Bailey, D. A., Faulkner, R. A., Kowalski, K. C., \& McGrath, R. (1997). Measuring general levels of physical activity: Preliminary evidence for the Physical Activity Questionnaire for Older Children. Medicine and Science in Sports and Exercise, 29, 1344-1349

De Piero, A., Rodríguez-Rodríguez, E., González-Rodríguez, L. G. y López-Sobaler, A. (2014). Sobrepeso y obesidad en un grupo de escolares españoles. Revista Chilena de Nutrición, 41 (3), 264-271.https://dx.doi.org/10.4067/S0717-75182014000300006

Fajardo, E. y Arango, L. (2012). Prevalencia de sobrepeso y obesidad, consumo de alimentos y patrón de actividad física en una población de niños escolares de la Ciudad de Bogotá. Revista Facultad de Medicina, 20 (1). 101-106. Recuperado de: http://www.scielo.org.co/ scielo.php?script $=$ Sci arttext\&pid=S0121-52562012000100011

Hallal, P.C., Andersen, L.B., Bull, F.C., Guthold, R., Haskell, W. \& Ekelund, U. (2012). Global physical activity levels: surveillance progress, pitfalls, and prospects. The Lancet, 380 (9838), 247-257. doi: http://dx.doi.org/10.1016/S0140-6736(12)60646-1

Högstrom, G., Nordström, A., \& Nordström, P. (2014). High aerobic fitness in late adolescence is associated with a reduced risk of myocardial infarction later in life: a nationwide cohort study in men. European Heart Journal, 35 (44), 3133-3140 doi: https://doi.org/10.1093/eurheartj/eht527

Janz, K. F., Lutuchy, E. M., Wenthe, P. \& Levy, S. M. (2008). Measuring activity in children and adolescents using self-report: PAQ-C and PAQ-A. Medicine \& Science in Sports \& Exercise, 40 (4), 767-772. Recuperado de: https://www.ncbi.nlm.nih.gov/pubmed/18317366

Kowalski, K, Crocker, P.\& Faulkner R.A (1997). Validation of the Physical Activity Questionnaire for Older Children. Pediatric Exercise Science, 9, 174-186.

Lawrence, D. (1981). The delevolpment of a self-esteem questionnaire. Brithish Journal Educational Psychology, 51 (2), 245-251. Recuperado de: http://onlinelibrary.wiley.com/ doi/10.1111/j.2044-8279.1981.tb02481.x/abstract

López-Sánchez, GF., Borrego Balsalobre, FJ. \& Díaz Suárez, A. (2013). Effects of a physical activity program on body composition of school children of 3-5 years. SPORT TK: Revista Euroamericana de Ciencias del Deporte, 2 (2), 41-44.

López Sánchez, L., López Sánchez, G. F. \& Díaz Suárez, A. (2015). Effects of a physical activity program on the body image of schoolchildren with ADHD. Cuadernos de Psicología del Deporte, 15 (2), 135-142. doi: http://dx.doi.org/10.4321/S1578-84232015000200015

López-Sánchez, GF., Nicolás-López, J. \& Díaz-Suárez, A. (2016). Effects of a program of intense physical activity on the body composition of adolescents from Murcia. SPORT TK: Revista Euroamericana de Ciencias del Deporte, 5 (2), 83-88. 
López-Sánchez, GF., González-Víllora, S. \& Díaz-Suárez, A. (2016). Level of habitual physical activity in children and adolescents from the Region of Murcia (Spain). SpringerPlus, 5 (1015). doi: http://dx.doi.org/10.1186/s40064-016-2033-8

López -Sánchez, G. F., Ahmed, D., Borrego-Balsalobre, F. J., López-Sánchez, L. \& Díaz Suárez, A. (2016). Level of habitual physical activity in 8-9 years old schoolchildren from Spain and India. MHSalud: Revista en Ciencias del Movimiento Humano y Salud, 12 (2), 1-10.

Martínez-Gómez, D., Martínez-de-Haro, V., Pozo, T., Welk, G. J., Villagra, A., Calle, M. E., Marcos, A. y Veiga, O. L. (2009). Fiabilidad y validez del cuestionario de actividad física PAQ-A en adolescentes españoles. Revista Española de Salud Pública, 83 (3), 427-439. doi: http://dx.doi.org/10.1590/S1135-57272009000300008

Meredith, M. D.\&Welk, G. J. (2010). Fitnessgram \& Activitygram Test administration manual ( $4^{\text {ta }}$ ed.). Champaign, IL: Human Kinetics.

Mancipe, J. A., García, S. S., Correa, J. E., Menéses, J. F., González, E., y Schmidt, J. (2015). Revisión Efectividad de las intervenciones educativas realizadas en América Latina para la prevención del sobrepeso y obesidad infantil en niños escolares de 6 a 17 años: una revisión sistemática. Nutrición Hospitalaria, 31 (1) 102-114. Recuperado de http://www. redalyc.org/articulo.oa?id $=309232878010$

Mérida, R., Serrano, A. y Tabernero, C. (2015). Diseño y validación de un cuestionario para la evaluación de la autoestima en la infancia. Revista de Investigación Educativa, 33(1), 149162.http://revistas.um.es/rie/article/viewFile/182391/170841

Moore, J. B., Hanes, J. C. Jr, Barbeau, P., Gutin, B., Treviño, R. P. \& Yin, Z. (2007). Validation of the Physical Activity Questionnaire for Older Children in children of different races. Pediatric Exercise Science, 19(1), 6-19.

Morera-Castro, M., Jiménez-Díaz, J., Araya-Vargas, G. y Herrera-González, E. (s.f.). Cuestionario Pictórico de Actividad Física Infantil: diseño y validación. Sin publicar.

Organización Mundial de la Salud [OMS] (2010). Recomendaciones mundiales sobre actividad física para la salud. Ginebra, Suiza: OMS.

Organización Mundial de la Salud [OMS] (2015a). El método STEPwise de vigilancia. Recuperado de: http://www.who.int/chp/steps/es/

Organización Mundial de la Salud [OMS] (2015b). Obesidad y sobrepeso. Nota descriptiva N.311 Recuperado de: http://www.who.int/mediacentre/factsheets/fs311/es/

Ortega, M., Muros, J. Palomares, J. Martín, J. y Cepero, M. (2014). Influencia del índice de 
masa corporal en la autoestima de niños y niñas de 12 a 14 años. Asociación Española de Pediatría. 83 (5). doi: http://dx.doi.org/10.1016/j.anpedi.2014.11.017

Raimann, X. y Verdugo, F. (2012). Actividad física en la prevención y tratamiento de la obesidad infantil. Revista Médica Clínica Las Condes, 23 (3), 218-225. doi: http://dx.doi.org/10.1016/ $\underline{\text { S0716-8640(12)70304-8 }}$

Rivera, J., González, T., Pedraza, L., Aburto, T., Sánchez., T, \&Martorell, R. (2014). Childhood and adolescent overweight and obesity in Latin America: a systematic review. Lancet Diabetes Endocrinol, 70 (13), 173-6. doi: http://dx.doi.org/10.1016/s2213-8587(13)70173-6

Romero, E., Márquez-Rosa, S., Bernal, F., Camberos, N., De Paz, J.A. (2014). Imagen corporal en niños obesos: efectos producidos por un programa de ejercicio físico. Revista de Ciencias del Deporte, 10 (3), 149-162.

Trescastro-López, S., Trescastro-López, E. \& Galiana-Sánchez, M. E. (2014). A bibliometricstudy of food and nutrition education programmes and interventions in schools in Spain. Revista Española de Nutrición Humana y Dietética,18 (4), 184-193.

Trujano, P., Nava, C., de Gracia, M., Limón, G., Alatriste, A., y Merino, M. (2010). Trastorno de la imagen corporal: Un estudio con preadolescentes y reflexiones desde la perspectiva de género. Anales de Psicología, 26 (2), 279-287. Recuperado de http://www.redalyc.org/ articulo.oa? id $=16713079012$

Tsang, T.W., Kohn, M.R., Chow, C.M. \& Singh, M.F. (2013). Self-perception and attitude toward physical activity in overweight/obese adolescents: the "martial fitness" study. Res Sports Med., 21(1), 37-51. doi: https://dx.doi.org/10.1080/15438627.2012.738444

Ureña, P., Araya, F., Sánchez, B., Salas, J.\& Blanco Romero, L. (2010). Perfil de calidad de vida, sobrepeso-obesidad y comportamiento sedentario en niños (as) escolares y jóvenes de secundaria guanacastecos. Revista Electrónica Educare, XIV (2), 207- 224. Recuperado de http://www.redalyc.org/articulo.oa?id=194115606015

Welk G. J., Going S. B., Morrow J. R. Jr.\& Meredith M. D. (2011). Development of new criterionreferenced fitness standards in the FITNESSGRAM program: Rationale and conceptual overview. American Journal of Preventative Medicine, 41 (4), S63-S67. doi: http://dx.doi. org/10.1016/j.amepre.2011.07.012

Zhu W., Mahar M. T., Welk G. J., Going S. B., Cureton K. J. (2011). Approaches for development of criterion-referenced standards in health-related youth fitness tests. American Journal of Preventative Medicine, 41, S68-S76. doi: http://dx.doi.org/10.1016/j.amepre.2011.07.001 

de Investigación en Salud. España: Editorial Universidad de Almería.

Recepción: 8 de agosto del 2016

Corrección: 10 de enero del 2016

Aceptación: 21 de enero del 2016

Publicación: 31 de enero del 2017

1 Licenciado en Ciencias del Deporte con énfasis en Salud (UNA). Maestría en Psicopedagogía (UNED). Ha sido capacitador para Centroamérica y Ecuador del "Programa Apúntate a Jugar" desde el año 2008. Actualmente, es académico en la Escuela de Ciencias del Movimiento Humano y Calidad de Vida de la Universidad Nacional y coordina el Programa Psicomotricidad Infantil en la misma Unidad Académica.

2 Ph.D en Kinesiología con énfasis en comportamiento motor de la Universidad de Auburn, Estados Unidos de América. Máster en Recreación en la Universidad de Costa Rica. Actualmente académica, investigadora y extensionista en la Escuela de Ciencias del Movimiento Humano y Calidad de Vida, Universidad Nacional. Forma parte del grupo de trabajo del laboratorio de Psicomotricidad Infantil de CIEMHCAVI, Universidad Nacional.

3 Magister Scientiae en Ciencias del Movimiento Humano por la Universidad de Costa Rica. . Desde el año 2000 labora como docente e investigador en la Escuela de Educación Física y Deportes de la UCR y en la Escuela de Ciencias del Movimiento humano y Calidad de Vida (en ese entonces, Escuela de Ciencias del Deporte) de la UNA. Cuenta con varias publicaciones en el campo de la educación física, de la psicología del ejercicio y el deporte, así como en el ámbito de la salud y del rendimiento deportivo.

4 Bachiller en Psicología Universidad de Costa Rica, Licenciado en Psicopedagogía, Universidad Nacional, Master Scientae en Salud Integral y Movimiento Humano, Universidad Nacional. Psicólogo y psicopedagogo socio fundador del Instituto Latinoamericano de Psicología y Pedagogía Alternativas ILPPAL, Rio Oro de Santa Ana. En la actualidad participa como docente universitario en la Escuela de Ciencias del Movimiento Humano y Calidad de Vida, UNA y como psicólogo y psicopedagogo del Programa de Investigación Psicomotricidad Infantil, PSICOMI, UNA.

5 Licenciada en Ciencias del Movimiento Humano con énfasis en Rendimiento Deportivo. Universidad Nacional. Licenciada en Terapia Física. Universidad Americana. Educadora física del programa de investigación Psicomotricidad e Intervención y Docente Escuela Ciencias del Movimiento Humano y Calidad de Vida. Entrenadora del equipo representativo femenino de Fútbol Sala de la Universidad Nacional. Vicerrectoría de Vida Estudiantil. Departamento de Promoción Estudiantil. UNA. Preparadora física de la Selección Nacional de Futsal Femenino. FEDEFUTBOL. 